\title{
DUAL RESONANCE MODELS AND SUPERSTRINGS
}


This page is intentionally left blank 


\section{DUAL RESONANCE MODELS AND SUPERSTRINGS}

\section{Paud H Frampton}

University of North Carolina at Chapel Hill

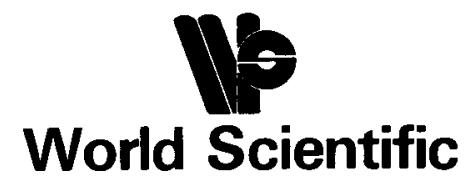




\author{
Published by \\ World Scientific Publishing Co Pte Ltd. \\ P. O. Box 128, Farrer Road, Singapore 9128.
}

Thanks are due to the authors, the American Physical Society and NorthHolland Publishing Co. for permission to reproduce the articles reprinted in this volume.

Library of Congress Cataloging-in-Publication data is available.

\title{
DUAL RESONANCE MODELS AND SUPERSTRINGS \\ Copyright $\odot 1986$ by World Scientific Publishing Co Pte Ltd.
}

All rights reserved. This book, or parts thereof, may not be reproduced in any form or by any means, electronic or mechanical, including photo. copying, recording or any information storage and retrieval system now known or to be invented, without written permission from the Publisher.

ISBN 9971-50-080-9

9971-50-081-7 (pbk)

Printed in Singapore by Kyodo-Shing Loong Printing Industries Pte Ltd. 


\section{PREPACE TO REPRINTED AND \\ EXPANDED EDITION}

\section{The development of string and superstring theories has an}

interesting history malnly because the perlods of intense research activity 1968-74 and 1984- are separated by some ten years during which only a small number of papers were published. "Dual Resonance Models" was the only book on dual models and strings published during the first period. Shortly after its publication, the bottom dropped out of the fleld. The book went out of print in 1979, by which time interest in the subject had waned.

The main reason for reprinting the 1974 text of "Dual Resonance Models" is the widespread resurgence of interest in string theories. The book provides a comprehensive set of references for the period 1968-74 and, beyond that, many of the 1deas and techniques are still germane in present superstring theories. A Supplement on superstrings, together with a small set of reprinted papers, has been added; hence the new title, "Dual Resonance Models and Superstrings." In the reprinted text from "Dual Resonance Models," typographical errors have been corrected and references updated.

As a digression, my first intimation that something unexpected had occurred in string theory (after ten years) came while visiting CERN, Switzerland, In August 1984, when Lars Brink told me from Michael Green and John Schwarz, who were then at the Aspen Center for 
Physics, Colorado, that $O(32)$ is anomaly free for open superstrings. This message was conveyed to me because just four months earlier I had stated at the ICTP in Trieste, Italy, that all open superstrings were potentially anomalous; Green and Schwarz had been In the audience. Now they had discovered the elegant mechanism by which $O(32)$ uniquely escapes the fatal difficulty of chiral anomalies.

The construction of the $\mathrm{E}(8) \times \mathrm{E}(8)$ heterotic string by David Gross and his collaborators at Princeton University gave a more geometrical scheme for introduction of Yang-Mills symmetry than the unsatisfying multiplicative Chan-Paton factor of old. These developments compelled the sclentific community to take seriously the Idea of a superstring as a "theory of everything." Firstly, it is a real candidate for a consistent model of quantum gravity; the theory not only can describe gravity but actually requires that gravity must exist. Second, by compactification to four spacetime dimensions one can hope to make contact with the gauge field theorles which successfully describe strong and electroweak interactions at experimentally accessible energies.

of course, there is room for skepticism. Superstrings have no basis in, or even motivation from, experiment. Further, superstrings seem to have been discovered by historical accident and therefore may be just one of many possible consistent frameworks for quantum gravity. Certainly experiment, and not just mathematical consistency, Is the ultimate test of any physical theory. Nevertheless, it is when one makes detalled calculations in superstring theory that one is constantly impressed by the remarkable properties which emerge. Superstrings could provide at least an existence theorem for a finite quantum gravity unified with gauge fleld theory.

The abrupt 1974 demise of dual resonance models for strong interactions was foreseeable because an alternative approach, gauge field theory, had been potentially available for almost twenty years. An 
equally abrupt demise of superstrings would require an alternative, better approach to quantum gravity. At present, to my knowledge, a plausible competitor to the superstring is nowhere in sight. At this time, therefore, the superstring is expected to remain of interest to physicists for several years; it seems likely that it will take that long to establish any meaningful confrontation with experiment. If and when any agreement between superstrings and experiment is found, it will certainly justify an entirely new book on the subject.

I wish to thank Professor J. C. Pati for encouragement to reprint and expand "Dual Resonance Models." The Benjamin/Cumnings Publishing Company generously assigned and transferred its 1974 copyright. I also thank Professor T. Kobayashi for the opportunity to give lectures on the superstrings Supplement at Tokyo Metropolitan University in April 1986. Finally, I thank Jody Gollan for typing the new cameraready material. 
This page is intentionally left blank 
ORIGINAL PREFACE*

Despite the fact that local field theory is successful in describing electromagnetic, and to a lesser extent, weak interactions, it seems to be plagued with difficulties when applied to strong interactions. Firstly, the avaflable methods in fleld theory are largely tied to perturbation expansions in the coupling constant and the coupling constant for strong interactions is large; secondly, because of the proliferation of strongly-interacting particles it seems impracticable to associate a new fleld to each new particle. Because of these problems, the S-matrix approach was developed which rejects the field as the important concept and attempts to study directly the transition matrix elements. This work is based on the fundamental postulates of (1) Polncare Invariance, (2) Cluster decomposition, (3) Analyticity, (4) Unitarity and (5) Crossing symmetry. The main difficulty here lies in disentangling the complicated non-linear relations implied by unitarity; in general, rather drastic simplifying assumptions must be made.

*To "Dual Resonance Models" (Benjamin, 1974). 
During the last few years, however, some new light has been shed on the S-matrix approach through the introduction of the dual1ty 1dea, and that is our present subject.

As 18 well known, the concept of duality in strong interactions was first arrived at in 1967 by Dolen, Horn and Schnid from the study of the constraints inposed by analyticity and crossing symetry through the technique of finite-energy sum rules. It was found that the direct-channel resonances and the cross-channel Regge poles provided, in an average sense, equivalent descriptions of the same phenomena.

The notion of duality received its first precise formulation with the advent of dual resonance models, starting from Veneziano's proposal in 1968 of the Euler B function model for the four-point function. This model demonstrated how the direct-channel and crosschannel descriptions can be precisely equivalent for a sum over an infinite number of resonances. The presentation of this model clarified this question and precipitated a great deal of significant progress. First it was found that the model could be straightforwardly extended to a multiparticle amplitude embodying the same principles, and, more remarkably, that the resultant amplitude was completely factorlsable on a finite degeneracy. Secondly, towards the end of 1969 Virasoro pointed out that for a particular intercept value there were sufficient gauge relations to allow the possibility of eliminating indefinite-metric ghosts, although the complete proof that this Indeed happens was not arrived at until 1972.

The model thus exhibits enormous mathematical consistency: of course this simplest model is far from describing Nature-for example, the mass spectrum is quite unrealistic and there are no fermions.

There followed a search for more complicated and improved models. In 1971, Ramond, Neveu, and Schwarz developed a dual theory with several advantages over the earlier one. It added additional 
degrees of freedom, and enlarged the gauge algebra, In such a way that ghosts were st11l absent, and fermions could be included in a consistent way. Subsequently, a variety of methods have been used to look for an even better model but although proposals exist no one has yet demonstrated that his particular model satisfies all the required postulates.

Despite the lack of realism, the construction of these mode1s represents a significant advance in the S-matrix approach to strong interactions. It teaches us that we may try to satisfy the basic postulates in a resonance approximation, with the advantage that the implications of unitarity are greatly simplified for resonance exchange.

What follows has been developed out of lectures given at the Nordic School in Spötind, Norway (January 1972), at Bielefeld University, West Germany (Autumn 1972), at Syracuse University, New York (Spring 1973) and at the Ettore Majorana School in Erice, Sicily (July 1973). The material has been up-dated, approximately to April 1974.

In Part I we introduce the phenomenological concept of duality after giving some elementary discussion of Regge poles and resonances. This explains the motivation for constructing the dual resonance mode1s. Part II deals with the Venezlano function, its multiparticle generalisation and derives the exponential degeneracy of states. Here the very important projective group $0(2,1)$ is first introduced. In Part III the operator formulation of the model is analysed, making extensive use of the projective group. The no-ghost theorem is proved here.

The treatment of internal symmetry, particularly 1sospin, is made in Part IV. The difficulty of introducing broken symmetries (such as $\mathrm{SU}_{3}$ ) is pointed out, and then the rubber string derivation of the Veneziano model is given. The main subject of Part $V$ is the 
introduction of fermions, and the principal properties of the NeveuSchwarz-Ramond theory are worked through in some detall. In Part VI the symmetric group approach is used to classify the earlier models, and to lead the way towards improved ones. To correct, at least partially, for our theoretical bias we outline in Part VII some of the phenomenological applications of the generalised Euler B function formula. Finally, in an Appendix, we show how, in the linit of small Regge slope, dual resonance models reduce to lagrangian fleld theories.

Throughout, we give fairly complete derivations for all the algebraic and group theoretic results and only in a few less important cases are results cited without proof.

Over the last several years I have benefited in my knowledge of dual resonance models from interactions with many other theorists. A partial list Includes: D. Amati, L. Brink, R. C. Brower, P. G. 0. Freund, S. Fubini, M. Gell-Mann, P. Goddard, M. Jacob, Z. Koba (deceased), C. Lovelace, S. Mandelstam, Y. Nambu, A. Neveu, H. B. Nielsen, D. I. Olive, P. Ramond, R. J. Rivers, J. Scherk, C. Schmid, J. H. Schwarz, G. Veneziano and M. A. V1rasoro. I am grateful to these, and others, for enlightenment.

Finally it is a pleasure to thank Mrs. Joyce McManus, Mrs. Betty Osborne, Frau Irmela Schmidts and Mrs. Marjorle Warner for typing the manuscript. 


\section{TABLE OF CONTENTS}

Preface to Reprinted and Expanded Edition v

Original Preface $\quad$ ix

Part One: DUALITY

1.1 Introduction 1

1.2 Definitions and Kinematics 2

1.3 Single Variable Dispersion Relations 10

1.4 Resonances and Regge Poles 13

1.5 Superconvergence and Finite Energy Sum Rules 22

1.6 FESR Duality; Schmid Loops 26

1.7 Harari-Freund Ansatz 33

1.8 Exchange Degeneracy 34

1.9 Duality Diagrams 37

$\begin{array}{lll}1.10 & \text { The Situation of Mid-1968 } & 41\end{array}$

1.11 Multiparticle Production 45

1.12 Summary 50

References 51

$\begin{array}{lll}\text { Part } & \text { Two: MULTIPARTICLE DUAL MODEL } \\ 2.1 & \text { Introduction }\end{array}$

2.2 Veneziano's Beta Function and Its Properties 56

2.3 Five-Point Function 67

2.4 N-Point Function $\quad 71$ 
xiv

CONTENTS

2.5 Koba-Nielsen Form and Projective Invariance 77

2.6 Operator Factorisation and Level Density 83

2.7 Satellite Terms 90

2.8 Amplitude for Pion-Pion Scattering 95

2.9 Shapiro-V1rasoro Model 99

$\begin{array}{ll}2.10 & \text { Summary } \\ & 104\end{array}$

$\begin{array}{ll}\text { References } & 106\end{array}$

Part Three: OPERATOR FORMALISM

$\begin{array}{lll}3.1 & \text { Introduction } & 109\end{array}$

3.2 Projective Group $\quad 110$

3.3 Gauge Invarlance 122

$\begin{array}{lll}3.4 & \text { Operatorfal Duality } & 129\end{array}$

3.5 Untt Intercept 136

$\begin{array}{lll}3.6 & \text { Null States } & 142\end{array}$

$\begin{array}{lll}3.7 & \text { Critical Dimension } & 148\end{array}$

$\begin{array}{lll}3.8 & \text { Physical State Construction } & 157\end{array}$

3.9 Twisting Operator 165

3.10 Multireggeon Vertex 171

$\begin{array}{lll}3.11 & \text { Summary } & 179\end{array}$

$\begin{array}{ll}\text { References } & 181\end{array}$

Part Four: INTERNAL SYMMTRY

4.1 Introduction $\quad 184$

4.2 Multiplicative Internal Symmetry Factor 185

4.3 Factorisation of Unequal Intercepts 190

4.4 The Rubber String Model 194

4.5 Baryons and Exotics , 207

4.6 More on the Rubber String 219

4.7 Summary 234

$\begin{array}{ll}\text { References } & 236\end{array}$

Part Five: SPIN

5.1 Introduction 
5.2 Multiplicative Spin Factor 239

5.3 Free Fermions (Ramond) 247

5.4 Dual Plon Model (Neveu and Schwarz) 257

5.5 Spectrum 266

5.6 Boson-Fermion Couplings 275

5.7 Generalised Projective Invariance 284

5.8 Non-Planar Extension 291

5.9 Summary 293

References $\quad 295$

Part S1x: SYMMETRIC GROUP

6.1 Introduction 298

6.2 Methods of Attacking the Intercept Problem 299

6.3 Four-Meson Amplitude; Symmetric Group 301

6.4 Regge Behaviour and Analytic Properties 306

6.5 Explicit Construction 309

6.6 Four-Pion Amplitude $\quad 315$

6.7 Possible Connection to Three-Quark Baryons 322

6.8 Multiparticle Extension 325

6.9 Classification of Dual Resonance Models 329

6.10 Multipion Amplitude $\quad 334$

6.11 Spin-Lowering Symmetry 339

6.12 Factorisation 342

6.13 Summary 356

$\begin{array}{ll}\text { References } & 358\end{array}$

Part Seven: PHENOMENOLOGICAL APPLICATIONS

7.1 Introduction 362

7.2 Baryon-Antibaryon Annihilation 364

7.3 Meson-Nucleon Scattering 368

$7.4 \quad B_{5}$ Phenomenology 375

7.5 Single-Particle Inclusive Spectra 383

7.6 Many-Particle Inclusive Spectra 391 
xv1

7.7 Summary

392

References

394

Appendix: 2ERO-SLOPE LIMIT

A.1 The $\lambda \phi^{3}$ Limlt

398

A.2 Yang-Mills Fleld Theory 401

A.3 Regge Slope Expansion $\quad 410$

$\begin{array}{ll}\text { References } & 414\end{array}$

Supplement: SUPERSTRINGS

S.1 Introduction $\quad 415$

S.2 Gravitation $\quad 417$

S.3 Ten-Dimensional Supersymmetry $\quad 424$

S.4 Non-String Kaluza-Klein Theory 427

S.5 Anomaly Cancellation $\quad 440$

S.6 One-Loop Finiteness; Modular Invariance for Closed Strings 446

$\begin{array}{lll}5.7 & H e t e r o t i c ~ s t r i n g & 475\end{array}$

S.8 Superstring Phenomenology 486

$\begin{array}{lll}\text { S.9 Summary } & 499\end{array}$

$\begin{array}{ll}\text { References } & 500\end{array}$

Reprints:

1. P. H. Frampton and T. W. Kephart, "Bxplicity Evaluation of Anomalies in Higher Dimenstons," Phys. Rev. Lett. 50, 1343-1346 (1983). 507

2. M. B. Green and J. H. Schwarz, "Anomaly Cancellations in Supersymmetric D = 10 Gauge Theory and Superstring Theory," Phys. Lett. 149B, 117-122 (1984).

3. P. G. O. Freund, "Superstrings from 26 D1menstons?" Phys. Lett. 151B, 387-390 (1985).

4. D. J. Gross, J. A. Harvey, E. Martinec and R. Rohm,

"Heterot1c String," Phys. Rev. Lett. 54, 502-505 (1985). 